\title{
Review: group based parenting programmes improve short term mental health in infants and toddlers
}

\author{
Barlow J, Parson J. Group-based parent-training programmes for improving emotional and behavioural adjustment in \\ 0-3 year old children. Cochrane Database Syst Rev 2002;(2):CD003680 (latest version 2 Apr 2001).
}

\section{QUESTION: Does a group based parent training programme improve the short term mental health of children under 3 years of age?}

\section{Data sources}

Studies were identified by searching Medline (19702001), EMBASE/Excerpta Medica (1974-2001), Biological Abstracts (1985-2001), British Nursing Index (1994-2001), CINAHL (1982-2001), PsycINFO (19702001), Sociological Abstracts (1963-2001), Social Science Citation Index (1994-2001), ASSIA, the Cochrane Library (Issue 3, 2001), the National Research Register (Issue 4, 2001), Dissertation Abstracts (1980-2001), ERIC, and bibliographies of relevant papers.

\section{Study selection}

Randomised or quasi-randomised controlled trials were selected if they compared a group based parenting programme with a waiting list or no treatment control, involved parents of children aged $0-3$ years, and measured child mental health on $\geq 1$ standardised instrument.

\section{Data extraction}

Data were extracted on study quality, participants, interventions, and outcomes. Authors were contacted to obtain missing data.

\section{Main results}

5 studies of behavioural or cognitive behavioural parenting group programmes were included (190 participants, involving 36 assessments of infant and toddler mental health). 4 studies (102 participants, 52 in intervention and 50 in waiting list control groups) reported on 28 measures of child emotional and behavioural adjustment; 4 of these measures were included in a meta-analysis (random effects model) that showed a postintervention result favouring the intervention (standardised mean difference [SMD] $-0.58,95 \%$ CI -1.39 to -0.22 ). Of these 4 studies, 1 study (8 wk behavioural programme) showed that the intervention improved child behaviour (Child Behaviour Questionnaire, SMD -1.5 , CI -2.6 to -0.46 ) and behaviour in home situations (Home Situations Questionnaire, SMD -1.34 , CI -2.37 to -0.31 ), and another study (4 cognitive behavioural training sessions) showed that the intervention improved child emotional and behavioural adjustment (Behavioural Screening Questionnaire, SMD -0.8, CI -1.44 to -0.1$)$. For these 4 studies, follow up ranged from 1-18 months; in 3 studies, data were unavailable for both intervention and control groups and in 1 study, the intervention improved some child behavioural Source of funding: PPP Healthcare Trust $U K$.

For correspondence: Dr J Barlow, University of Oxford, Oxford, UK. outcomes at 3 months. 1 study (4 behavioural training sessions) that focused on improving sleep patterns in newborn infants showed that group training improved the number of sleep episodes, duration of the longest sleep episode, and numbers sleeping >300 minutes immediately after intervention and at 4 months, but did jane.barlow@dphpc.ox.ac.uk not affect total sleep.

\section{Conclusions}

Group based parenting programmes improve short term emotional and behavioural adjustment at $<18$ months, and sleep patterns in children $<3$ years of age. Data are unavailable to show the long term effectiveness of these programmes to maintain improvements in child mental health and to prevent subsequent development of mental health problems in very young children.

\section{COMMENTARY}

Since the 1960s when Gerald Caplan first initiated the concept of a population focused approach to enhance the mental health of children, ${ }^{1}$ research has shown the effect of parenting on mental health in children and its predictive value for the development of mental illnesses in adulthood. Based on recent data suggesting that the incidence of emotional and behavioural problems in children is beginning to exceed treatment resources, ${ }^{23}$ Barlow et al identified the need for evidence-based interventions to address the problem.

The authors' choice of a systematic review and metaanalysis as their study design has major strengths. The investigators were careful to include studies that met specific criteria. Barlow et al described a detailed selection process to ensure such validity. The systematic review showed that several studies from different places are consistent in their finding of a positive effect of parenting programmes. Combining data from several studies in a meta-analysis enhances the power of the analysis and the strength of the conclusions. Study limitations include small sample sizes of individual studies, inclusion of quasi-randomised studies, and variations in the group interventions and follow up data.

The results of this systematic review are relevant to nurse practitioners in the mental health field and to public health nurses. The findings provide evidence that nurses confronted by both an increased need for service and a shortage of service providers can partially close the gap by using a group based parenting programme to address the emotional and behavioural adjustment of children $<3$ years of age. However, this review did not compare and contrast specific types of group based parenting programmes. Further studies are needed to show the effect of specific group based parenting programmes. Research is also needed to examine the long term effects of treatment programmes and to study approaches to prevent mental health problems in children. Lynda Slimmer, RN, PhD Research Subject Advocacy Program Coordinator General Clinical Research Center University of Illinois Chicago, Illinois, USA

1 Caplan G. Prevention of mental disorders in children; initial explorations. New York: Basic Books, 1961.

2 Campbell SB. Behavior problems in preschool children: a review of recent research. J Child Psychol Psychiatry 1995;36:113-49

3 Department of Health. Improving the health of mothers and children: NHS priorities for research and development. Report to the NHS Central Research and Development Committee. NHS Executive. Leeds, 1995. 